\title{
Callyntra hibrida n. sp.: Un nuevo tenebriónido de Chile central (Coleoptera: Tenebrionidae)
}

\section{Callyntra hibrida n. sp.: A new darkbeetle from central Chile (Coleoptera: Tenebrionidae)}

\author{
Álvaro Zúñiga-Reinoso ${ }^{1} \&$ Mauricio CID-Arcos ${ }^{2}$ \\ 1Programa de Doctorado en Ciencias mención Ecología y Biología Evolutiva, Facultad de Ciencias, Departamento de Ciencias \\ Ecológicas, Universidad de Chile. \\ *E-mail: alzure@gmail.com \\ ${ }^{2}$ Calle 5 Norte \#3833, Talca.
}

\begin{abstract}
RESUMEN
Callyntra Solier,1836, es un género endémico de Chile, que contiene 17 especies descritas hasta la fecha. En este artículo se describe a Callyntra hibrida, una nueva especie de tenebriónido de la cordillera de la Costa de Chile Central.

Palabras clave: Pimeliinae, Nyctelini, nueva especie, taxonomía.

\section{ABSTRACT}

Callyntra Solier, 1836, is a endemic genus from Chile, which contains 17 currently described species. In this paper, Callyntra hibrida, a new species of darkbeetle to Central Chile is described.
\end{abstract}

KeYwords: Pimeliinae, Nyctelini, new species, taxonomy.

\section{INTRODUCCIÓN}

El género Callyntra Solier, 1836, incluye a un conjunto de especies endémicas de Chile Central, pertenecientes a la tribu Nyctelini. Luego de una compleja historia taxonómica, se estableció como grupo natural, con una revisión y posterior revalidación del género Callyntra (Flores \& Vidal 2000a) y la validación de las relaciones filogenéticas entre las especies (Flores \& Vidal 2000b). De esta manera, este género presenta actualmente 17 especies descritas, distribuidas desde $32^{\circ}$ a los $39^{\circ}$ latitud sur, encontrándose en ambas cordilleras y terrazas costeras (Flores \& Vidal 2000a, 2000b). Hasta la fecha, no existen nuevas contribuciones que describan nuevas especies y menos aún, trabajos acabados relacionados con la ecología y biología de estos insectos. Al respecto Flores \& Vidal (2000a), mencionan que nada se conoce sobre el ciclo de vida de este género. Sólo se han descrito las larvas de dos especies del género y existen algunas observaciones de campo, donde se mencionan que las especies de este género son caminadoras, de hábitos principalmente diurnos (Flores \& Vidal 2000a, Vidal \& Guerrero 2007).

Recientemente se ha recolectado material de Callyntra proveniente del cordón montañoso costero de Altos de Cantillana, las que poseen características morfológicas externas únicas, que difieren del resto de las especies del género y que se describe detalladamente más adelante.

\section{MATERIALES Y METODOS}

El material fue recolectado de forma manual en los faldeos del cerro Poqui, entre los 600 y 800 m.s.n.m. y conservado en alcohol. Una parte del material tipo fue debidamente limpiado, montado en seco y etiquetado. Mientras que otra parte fue conservada en alcohol 99.8\%. Los ejemplares se encuentran depositados en las siguientes colecciones:

MNNC - Museo Nacional de Historia Natural, Santiago, Chile.

MZUC- Universidad de Concepción, Concepción, Chile. IADIZA - Instituto Argentino de Investigaciones de las Zonas Áridas, Mendoza, Argentina.

GEVOL - Laboratorio de Genética y Evolución, Facultad de Ciencias, Universidad de Chile.

CPMC - Colección Particular de Mauricio Cid. CPPP -Colección Particular de Pablo Pinto 


\section{CPSL -Colección Particular de Sebastián Larrea}

Por otra parte, para establecer las diferencias entre los taxa, se compararon las genitalia de los machos de los nuevos morfos, con las especies morfológicamente más cercanas, según lo propuesto por Flores (1996). Los dibujos fueron realizados y escalados utilizando el programa CorelDraw5x.

\section{RESULTADOS}

Callyntra hibrida Zúñiga-Reinoso \& Cid-Arcos n. sp. (Fig. 1) DESCRIPCIÓN:

Coloración: cuerpo negro, patas en general negras. Cabeza: con rugosidades; clípeo con 4 a 5 setas dispersas en las depresiones laterales. Margen lateral del clípeo con densa pilosidad, sin o leve puntación y escasas arrugas longitudinales, sutura clipeal no visible. Labro con setas en toda la superficie que se alargan hacia los bordes. Margen lateral del clípeo con densa pilosidad. Margen anterior del labro escotado en V. Frente glabra, con una protuberancia en el centro, del cual se origina una carina longitudinal hacia la parte posterior. Esta estructura es circunscrita por carinas menores y separando a ambas carinas una ranura profunda. Vertex con una figura solevantada en forma de pentágono. Mentón con setas largas y densamente punteado. Antenas de color negras con abundante pilosidad blanca, lo que las hace en apariencia aterciopelada, llegando hasta 3/4 del margen del pronoto. Tercer antenito más largo que el cuarto y decimoprimer antenito más estrecho que el noveno y décimo. Ojo con abundantes setas saliendo de la base.

TóRAX: Pronoto glabro, sin puntuación, ángulo anterior redondeado, margen anterior con puntuación. La parte central del margen anterior levemente ensanchado, estrechándose hacia los costados. Una ranura separa el margen anterior del disco, sin arrugas entre las estructuras. Margen lateral cóncavo en la mitad anterior, convexo en el centro donde se encuentra la punta de la proyección central del pronoto. La mitad posterior recta y estrechándose. Cinco o seis ranuras pequeñas transversales en $2 / 3$ parte del margen lateral. Ángulo posterior proyectado hacia atrás, por sobre los élitros, arrugas paralelas entre las carinas y rugosidades aleatorias en la mitad posterior entre la carina y el margen lateral del pronoto. Proepiesterno con pilosidad esparcida y agrupada en la parte central de la estructura, con estrías longitudinales marcadas. Proesterno convexo, semiarrugado y con setas esparcidas. Apófisis proesternal subrectangular distalmente expandida, con setas distribuidas longitudinalmente, ligeras arrugas en la superficie principal y estrías profundas en todo el margen. Mesoepisterno con setas distribuidas por todo el margen y sin estrías. Metaepisterno, liso, con setas distribuidas transversalmente por el margen posterior.

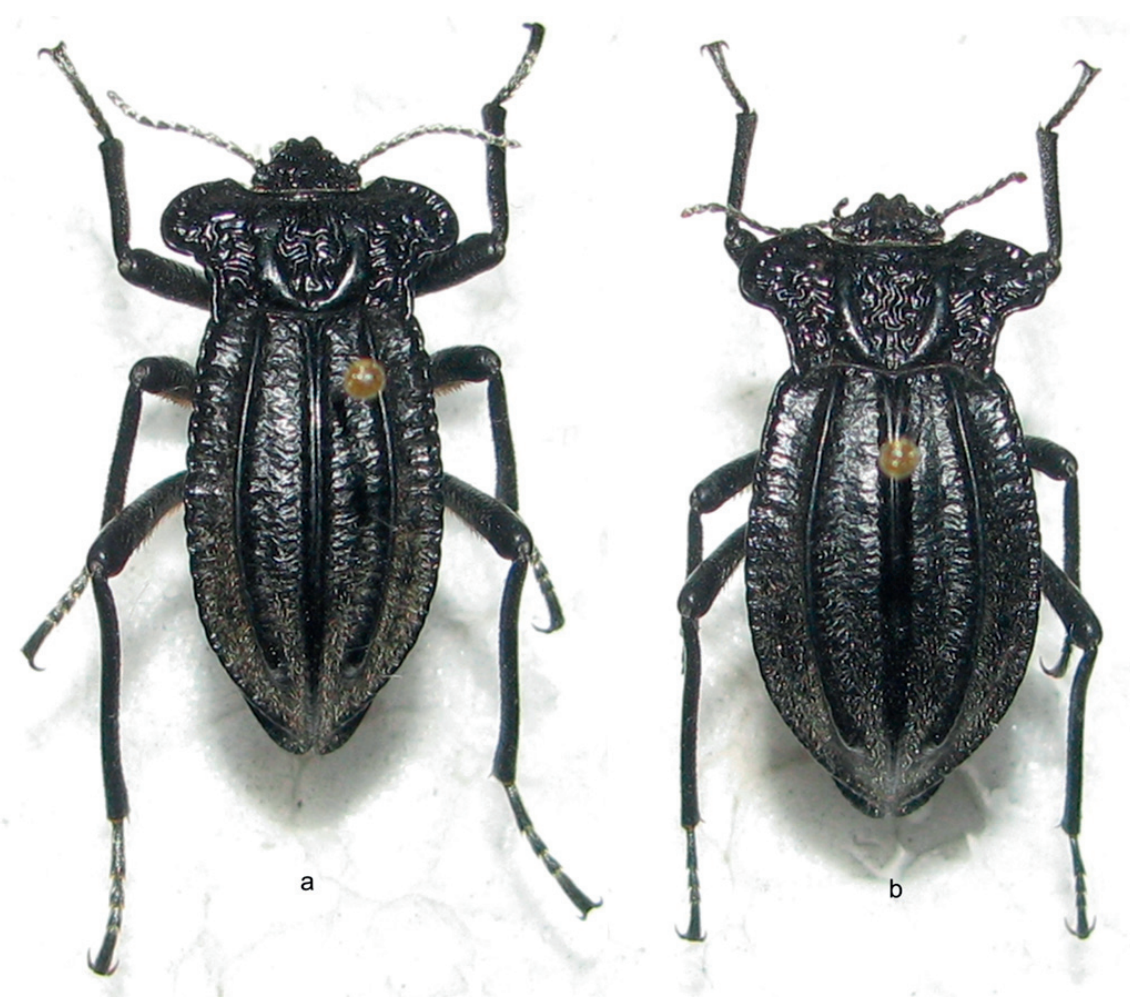

Figura 1. Fotografía en vista dorsal de Callyntra hibrida Zúñiga-Reinoso \& Cid-Arcos n. sp.: a) macho y b) hembra.

Figure 1: Photography in dorsal view of Callyntra hibrida Zúñiga-Reinoso \& Cid-Arcos n. sp.: a) male and b) female. 
ÉLitros: Primeros $2 / 3$ de los élitros glabro, pilosidad blanca esparcida en el último cuarto siendo más notorio en el declive elitral (principalmente alrededor de las carenas), con una costa en cada élitro, careciendo de costas secundarias. Sin embargo, en la mitad posterior de los élitros aparece una estructura solevantada parecida a una pseudocosta apenas notoria. Sutura elitral levantada. Espacios entre la sutura/costa y costa-margen con rugosidad transversa leve. Margen lateral notorio y crenulado. Epípleura glabra y lisa. Pseudopleura glabra, salvo en la parte terminal donde presenta algunas setas, con leve rugosidad que se acrecienta cerca de la epípleura.

ABDOMEN: Ventritos glabros y de coloración general negra brillante, con puntuación fina, escasa e irregular. Cuarto ventrito notoriamente más estrecho que el resto (1:2).

Patas: Todas las patas de color negro. Coxas glabras con puntuación irregular gruesa; trocánter con abundantes setas de coloración blanquecina de igual largo en todas las patas. Fémures con densa pilosidad blanca en su faz inferior, mientras que en su faz superior posee pilosidad blanquecina más corta y dispersa. Tibias en su faz inferior al igual que en los fémures, presenta pilosidad densa pero de menor tamaño (1:3), mientras que en su faz superior disminuye la pilosidad observándose una puntuación irregular gruesa. Tarsos con abundante pilosidad blanca que envuelve toda la estructura.

Genitalia: Edeago con los parámeros del tegmen cóncavos, que se agudizan hacia el ápice. Margen basal bisinuado, incompleto, formando un espacio romboidal. Presenta escasas setas cortas esparcidas en la primera mitad apical en la superficie ventral, las primeras aglomeradas en grupo de 3 o 4 setas. Láminas basales del tegmen con base redonda (Fig.2a). Al comparar la genitalia de C. hibrida, con la de otras especies de Callyntra morfológicamente cercanas, se puede ver que esta estructura difiere considerablemente con las otras especies, debido al mayor tamaño de la genitalia al compararla con Callyntra laticollis y debido a que posee los parámeros del tegmen menos curvados que los de Callyntra paulseni (Fig. 2b).

TAMAÑOS CORPORALES: Se entregan los tamaños mínimos y máximos observados de la serie tipo.

MAchos: largo total: $17,45 \mathrm{~mm}-19 \mathrm{~mm}$; ancho máximo pronotal: $8 \mathrm{~mm}-9 \mathrm{~mm}$; largo pronotal: $3,55 \mathrm{~mm}-4 \mathrm{~mm}$; largo elitral: $12,45 \mathrm{~mm}-13,7 \mathrm{~mm}$; ancho elitral: $7,2 \mathrm{~mm}-$ $7,7 \mathrm{~mm}$; altura élitros: $5,5 \mathrm{~mm}-6,6 \mathrm{~mm}$.

Hembras: largo total: $13,95 \mathrm{~mm}-20,60 \mathrm{~mm}$; ancho máximo pronotal: $8 \mathrm{~mm}-9,4 \mathrm{~mm}$; largo pronotal: $3,55 \mathrm{~mm}$ - 4,4 mm; largo elitral: 13,4 mm - 15,4mm; ancho máximo elitral: 7,7 mm - $10 \mathrm{~mm}$; altura élitros $5,75 \mathrm{~mm}-7 \mathrm{~mm}$.

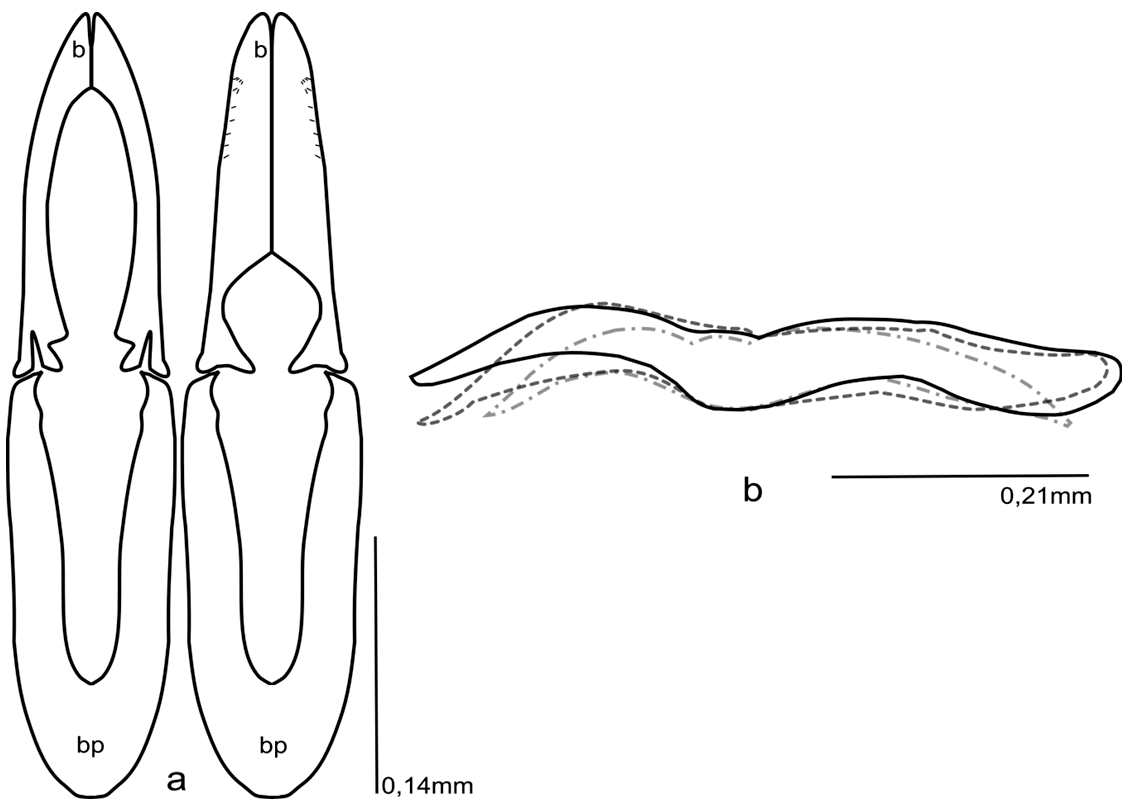

Figura 2. Genitalia de machos de Callyntra hibrida Zúñiga-Reinoso \& Cid-Arcos y comparación de genitalia con especies morfológicamente cercanas: a) Vista dorsal y ventral de la genitalia. bp: lamina basal del tegmen; p: parámeros del tegmen. b) Comparación de contornos en vista lateral de los aedeagos: línea continua negra: Callyntra hibrida, línea punteada gris oscura: Callyntra paulseni y línea punteada gris clara: Callyntra laticollis.

Figure 2. Male genitalia of Callyntra hibrida Zúñiga-Reinoso \& Cid-Arcos and genitalia comparison with species morphologically close: a) Dorsal and ventral views of the genitalia. bp: basal lamina of tegmen; p: parameres of tegmen. b) Contours comparison lateral view of aedeaga: solid black line: Callyntra hibrida, dark gray dotted line: Callyntra paulseni and light gray dotted line: Callyntra laticollis. 
VARIACIÓN INTRAESPECÍFICA: En general no se observó variación en toda la serie tipo de las estructuras detalladas en la descripción. Sólo se observó dimorfismo sexual en el tamaño, ya que las hembras poseen élitros más largos, altos $\mathrm{y}$ anchos que los machos.

HÁBitat: Todos los ejemplares han sido recolectados en el Cerro Poqui en la ladera con exposición sur, entre los $600 \mathrm{y}$ 800 m.s.n.m. Se encuentran en su mayoría asociados a los bordes de bosque de Nothofagus macrocarpa, caminando en lugares abiertos entre la hojarasca y senderos; incluso en laderas expuestas asociados a Puya sp.

ETIMOLOGíA: La nominación específica se debe a que morfológicamente parece un hibrido, ya que tiene aspecto de Callyntra paulseni debido a las expansiones laterales en el pronoto y a Callyntra penai por la forma de los élitros y las marcadas costillas.

Material examinado: Holotipo: Chile: Cachapoal: Doñihue, Rinconda de Parral, Cerro Poqui. 27. I.2013. col. A. Zúñiga, A. Fierro y J. Mondaca. Georeferencia: 3412'31'S; 71 3'33”O. 1 $0^{\Uparrow}$ (MNNC). Paratipos: Chile: Cachapoal: Doñihue, Rinconda de Parral, Cerro Poqui. 20. XII. 2012. col. M. Cid. 1ठ (CPMC). 2/3.I.2013. col. M. Cid. 25 ejemplares: $9 \hat{\jmath} / 7+$ (CPMC), $1 \lesssim / 1 q$ (MZUC: 40255-40256), $5 \hat{\jmath} / 2$ q (MNNC). 27.I. 2013. col. A. Zúñiga, A. Fierro y J. Mondaca. 4 ejemplares: $1 \hat{\jmath}$ (IADIZA), $2 \hat{\delta} / 1$ ㅇ (GEVOL). 31. X. 2013. col. P. Pinto. 10 ejemplares: $3{ }^{\lambda} /$ $3 q$ (CPPP), $2 \hat{\jmath} / 2$ ㅇ (MZUC: 40257 al 40260). 31. X/ 1.XI. 2013. col. S. Larrea. 10 ejemplares: $3 \hat{\jmath} / 3 \circ$ (CPSL), 1 ㅇ (MZUC: 40261), 1ふ/ 2ᄋ (IADIZA).

Distribución: Chile: Región de O’Higgins: Provincia de Cachapoal: Doñihue, Rinconda de Parral, Cerro Poqui.

\section{DISCUSION}

A partir de las observaciones morfológicas externas, se puede establecer que las características del pronoto, así como también de élitros y de la genitalia de los machos detalladas arriba, son caracteres constantes que permiten diferenciar a esta especie de las otras especies descritas (Fig 1 y 2 b). Por lo tanto, la especie que se describe, presenta características morfológicas (i.e morfología externa y genitalia) que permiten proponerla como una nueva especie, denominada aquí como $C$. hibrida. En su descripción, se incluyen las características morfológicas externas y la descripción de las estructuras del aedeago como lo ha propuesto Flores (1996), siendo este un carácter diagnóstico muy utilizado para diferenciar a las especies de la tribu y género (Peña 1994, Flores 1996, Flores \& Vidal 2000a, 2000b, Flores \& Carrara 2006). Los ejemplares observados no presentan variación intraespecífica considerable en las estructuras descritas, por lo que los caracteres observados en este trabajo son constantes en toda la serie tipo. Solo existe diferencia de tamaño entre los sexos, donde las hembras son más grandes que los machos, ya que presentan élitros más largos, altos y anchos, característica común en la familia Tenebrionidae de ambientes áridos y semiáridos (Cloudsley-Thompson 2001). Finalmente, la especie solo ha sido recolectada en la localidad tipo, en donde presenta bajas densidades poblacionales, por lo que consideramos que esta nueva especie endémica del Cerro Poqui, (cordón costero Alto de Cantillana), podría ser catalogada en algún estado precautorio de conservación.

\section{AGRADECIMIENTOS}

El primer autor agradece a Andrés Fierro, José Mondaca, Sebastián Larrea y Pablo Pinto por los consejos y el aporte de material que ha permitido el desarrollo de éste trabajo. A Moisés Villarroel por la ayuda en las capturas fotográficas de los aedegos. Además, el primer autor agradece especialmente a la beca CONICYT No 21110367 para estudios de doctorado. El segundo autor agradece a Jesús Bello por ser la compañía de tantas empresas de recolecta y a Mauricio Cid B. por haberlo iniciado en tan bella ciencia.

\section{BIBLIOGRAFIA}

Cloudsley-Thompson, J. 2001.Thermal and water relations of desert beetles. Naturwissenschaften 88: 447-460.

FLores, G.E. 1996. Estudio comparativo de las estructuras genitales en la tribu Nycteliini (Coleoptera: Tenebrionidae). Revista de la Sociedad Entomológica Argentina 55: 33-48.

Flores, G. \& R. Carrara. 2006. Two new species of Nyctelia Latreille (Coleoptera: Tenebrionidae) from western Argentinean high mountains. Annales Zoologici 56: 487495.

Flores, G. E. \& VidAL, P. 2000a. Revalidation and systematic revision of the Chilean genus Callyntra Solier (Coleoptera: Tenebrionidae). Annals of the Entomological Society of America 93: 1052-1075.

Flores, G.E. \& Vidal, P. 2000b. Cladistic analysis of the Chilean genus Callyntra Solier (Coleoptera: Tenebrionidae), with description of a new species. Journal of the New York Entomological Society 108: 187-204.

Vidal, P. \& Guerrero, M. 2007. Los Tenebriónidos de Chile. 1a edición. Ediciones Universidad Católica de Chile. Santiago, Chile. 478 pp.

Recibido: 03.05.13

Aceptado: 23.10.13 